\title{
Au sujet de Santa Cruz, por ejemplo, un film de Günter Schwaiger
}

En torno a «Santa Cruz, por ejemplo ", una película de Günter Schwaiger

\section{Günter Schwaiger}

\section{(2) OpenEdition}

12 Journals

\section{Édition électronique}

URL : http://journals.openedition.org/conflits/14193

DOI : $10.4000 /$ conflits. 14193

ISSN : $1777-5345$

Éditeur :

CCLS - Centre d'études sur les conflits lilberté et sécurité, L'Harmattan

Édition imprimée

Date de publication : 20 décembre 2008

ISBN : 1157-966X

ISSN : 1157-996X

\section{Référence électronique}

Günter Schwaiger, « Au sujet de Santa Cruz, por ejemplo, un film de Günter Schwaiger », Cultures \& Conflits [En ligne], 72 I hiver 2008, mis en ligne le 07 janvier 2009, consulté le 30 mars 2021. URL: http://journals.openedition.org/conflits/14193; DOI : https://doi.org/10.4000/conflits.14193

Ce document a été généré automatiquement le 30 mars 2021.

Creative Commons License 


\title{
Au sujet de Santa Cruz, por ejemplo, un film de Günter Schwaiger
}

\author{
En torno a "Santa Cruz, por ejemplo », una película de Günter Schwaiger
}

Günter Schwaiger

Ce film se structure autour d'une fouille dans le village de Santa Cruz, où, comme dans d'autres villages espagnols (d'où le nom du film "Santa Cruz, par exemple»), on a découvert une fosse commune datant de la période franquiste. Outre l'expérience même de l'exhumation, Günter Schwaiger a filmé les réactions des villageois et les témoignages de plusieurs personnes dont les proches ont été enterrés là, puis déterrés ${ }^{1}$. Ce film a par ailleurs été l'une des pièces essentielles de la vidéo-installation «Fosse commune » réalisée en partenariat avec Tomás Ruiz-Rivas ².

C\&C: Qu'est-ce qui t'a motivé à filmer les exhumations de Santa Cruz de Salcedo? Comment en es-tu venu à cette décision?

Günter Schwaiger : J'étais en train de travailler avec des amis sur un film qui portait sur les Autrichiens qui ont pris part aux Brigades internationales et nous avons fait un entretien avec Emilio Silva ${ }^{3}$. Il nous a parlé de l'ARMH et de son travail. Cela m'a énormément impressionné. Au final, Emilio nous a invités à assister à une exhumation à Burgos. Nous y sommes allés seulement pour le voir. Mais l'atmosphère, les gens, les émotions et l'importance du moment nous ont à ce point bouleversés que nous avons décidé de faire un film sur le sujet. Il y avait des gens qui, après soixante-dix années de silence, parlaient pour la première fois en public de l'assassinat de leurs parents, de leurs frères ou de leurs grands-parents. Je n'avais jamais rien vécu de semblable. Comme disait Emilio : «Quand une fosse est ouverte c'est comme une métaphore. Ce n'est pas seulement la terre qui s'ouvre, c'est aussi l'intérieur des gens, et un silence de soixante-dix ans est rompu.»

C\&C: Une scène du film a retenu mon attention. Jusque-là, on avait entendu plusieurs personnes parler, des proches, surtout, de personnes qui ont été fusillées et enterrées dans des fosses communes, mais aussi des voisins, des habitants du village, certains favorables aux exhumations, d'autres nettement moins. Soudain, un banc, quatre hommes sont assis, ils sont vieux, ils portent des cannes, on dirait qu'ils prennent le soleil. Tu t'approches et tu leur demandes ce qu'ils pensent des exhumations. Ils ne disent rien. Ils ne disent pas qu'ils 
sont contre. Ils ne disent rien. Rien de rien. Le film devient muet. Comment as-tu fait face à ce silence ? Que te suggère ce silence?

G. S. : J'en ai eu froid dans le dos, et c'est encore ma sensation lorsque j'y pense. Nous nous sommes approchés de ces quatre hommes parce qu'ils étaient toujours là, en train de nous regarder en silence. Leur réaction a été d'une extrême hostilité sans qu'un seul mot soit prononcé. Je crois que cette image (et de fait, beaucoup de gens y voient une métaphore des années de silence obligé) a une force symbolique importante. Après avoir semé la terreur et la peur, le régime a propagé le silence. Un silence puissant, imposant, qui s'est étendu comme une masse gluante dans tous les coins et recoins. Sur ces visages muets, on voit la culpabilité et la mauvaise conscience, mais aussi l'arrogance et la haine. Ces hommes savaient qu'à quelques mètres de là, la vérité qu'ils avaient essayé de cacher pendant tant de temps était en train d'être déterrée. Ils ne pouvaient l'assumer.

C\&C : A la fin du film, l'un des petits-enfants que l'on voit à plusieurs reprises (il s'agit d'un homme qui parle très sereinement, la quarantaine environ) et un très jeune garçon s'approchent du lieu où se trouvait la fosse. Le jeune garçon déroule alors un drapeau avec les sigles de l'UGT (Union générale des travailleurs) et le pose à même le sol. Le petit-fils dit alors quelque chose comme: "Mon grand-père n'a jamais imaginé ce qui allait lui arriver, mais il n'a jamais non plus imaginé que son arrière petit-fils allait lui rapporter le drapeau pour lequel on l'avait tué ». C'est, de mon point de vue, un thème crucial pour nos histoires latino-américaines et aussi, sans doute, pour beaucoup d'autres. La possibilité que l'on se donne aujourd'hui (ou non) de comprendre pleinement dans ce présent qui est le nôtre, pourquoi ils ont été tués (en l'occurrence, je fais référence aux militants des gauches latinoaméricaines sous les dictatures des années 1970). À partir de ta propre expérience autour de l'histoire espagnole, et en particulier de cet épisode des exhumations, cette question te semble importante? L'est-elle pour les gens que tu as rencontrés?

G. S. :C'est une question fondamentale, surtout si on fait de l'analyse un outil pour apprendre et éduquer. Je crois que les générations suivantes doivent savoir pourquoi et dans quelles circonstances quelqu'un a été assassiné. Ce n'est que de cette manière que l'on pourra donner de la valeur aux comportements et l'appliquer aux situations que l'on vit. En Espagne, il y a des groupes qui font des exhumations des revendications politiques et d'autres qui laissent aux proches le soin de choisir en toute intimité. Dans tous les cas, les associations informent sur les raisons pour lesquelles quelqu'un a été assassiné, sur son rôle ou sa charge politique et sa position sociale. Mais rares sont les proches des victimes à chercher réellement des raisons politiques. Ils n'osent quasiment jamais aller au-delà d'un sentiment d'injustice. Le franquisme a été au pouvoir pendant tant d'années qu'il a réussi à faire que toute une génération ait honte du passé politique de ses aïeux ${ }^{4}$. La plupart ne sait pas (ou ne veut pas savoir) dans quel parti ou dans quel syndicat militaient ses proches. Cette dépolitisation est très forte, elle est à mettre en relation à la guerre civile.

Mais c'est la génération des petits-enfants qui, par ailleurs, est à la tête de ce mouvement - qui n'a plus peur ni honte et commence à resituer ses proches dans un contexte politique. Cependant, il y a encore des tabous importants sur ce thème et beaucoup de personnes rencontrées et interviewées nous ont dit des choses comme : «Non, mon grand-père ne faisait pas de politique. On l'a tué parce que c'était quelqu'un de bien, parce qu'il y avait des jalousies, parce qu'il voulait le bien-être de tous $»$. 


\section{NOTES}

1. L'échange avec le cinéaste a été réalisé à distance (Madrid/Buenos Aires). Nous avions au préalable vu le film au Centre culturel espagnol de Buenos Aires.

2. Voir l'entretien avec Tomás Ruiz-Rivas http://www.conflits.org/index14183.html

3. Fondateur de l'Association pour la récupération de la mémoire historique.

4. Günter Schwaiger fait surtout référence à la génération intermédiaire entre les tués et les petits-enfants qui les cherchent. Voir, à ce propos, le témoignage de Javier Ortiz, dans le film de Marie-Paule Jeunehomme http://www.conflits.org/index14173.html

\section{AUTEUR}

\section{GÜNTER SCHWAIGER}

D’origine autrichienne, Günter Schwaiger est réalisateur et metteur en scène. Il réside en Espagne depuis 1991. Selon ses mots, il « centre son travail sur les thèmes de la solitude et l'incommunication dans une société aliénée ». Parmi ses travaux les plus récents, on peut citer $E l$ Paraíso De Hafner, réalisé en 2007 (voir : www.hafnersparadise.com) et donc Santa Cruz, por ejemplo (2005) réalisé en collaboration avec Hermann Peseckas (Beta SP, 65 min). Ce film a reçu en 2006 le prix des arts et de la culture de la ville de Salzburg. 